\title{
COMPORTAMIENTO ÉTICO DE LAS PEQUEÑAS EMPRESA. UN CASO EMPÍRICO
}

\author{
M. Ríos-Manríquez \\ Universidade de Guanajuato \\ mrm2000mx@gmail.com
}

Submetido 30/04/2016 - Aceito 16/09/2017

DOI: $10.15628 /$ holos.2017.4499

\section{RESUMEN}

El comportamiento ético es un factor clave en la Responsabilidad Social Corporativa o Empresarial. Siendo ideal que todo tipo de empresas diseñe estrategias, acciones y medidas éticas. Sin embargo las empresas de menor tamaño se enfrentan a una serie de carencias como recursos humanos, técnicos y financieros para desarrollarse. Por ello este artículo tiene por objetivo analizar la influencia del comportamiento ético en las pequeñas empresas de Celaya, Guanajuato, México. Analizando seis valores éticos: principios que guían la conducta de sus colaboradores, cumplimiento con la normatividad legal, competencia leal, equidad en Precio-calidad, apoyo al desarrollo de sus proveedores y colaboradores denuncian actos de corrupción. Con una muestra de 87 pequeñas empresas se realizó un análisis mediante tablas de contingencia, análisis de correlaciones a través de chi-cuadrado de Pearson. Los resultados muestran que independientemente de si la organización se considera Socialmente Responsable o no, visualizan en el comportamiento ético, un elemento dispensable en la forma de gestionar su organización, así como la forma en que deben de conducirse sus colaboradores, otorgando mayor énfasis al cumplimiento legal y a la Equidad en Precio-calidad. Resaltando que todos los valores éticos tratados en esta investigación se correlacionan positiva y significativamente con la Responsabilidad Social de las empresas independientemente de si se consideran o no Socialmente Responsables.

PALAVRAS-CHAVE: comportamiento ético, responsabilidad social empresarial, pequeña empresa, competencia leal, cumplimiento legal.

\section{ETHICAL BEHAVIOR OF SMALL ENTERPRISES. AN EMPIRICAL CASE}

\begin{abstract}
The ethical behavior is a key factor of the Enterprise Social Responsibility. Being ideal that every single company develops ethical strategies, actions and measures. However, smaller companies encounter several difficulties, such as lack of human, technical or financial resources, that limit the development of such ethical behaviors. This article has as its main goal to give a current picture of the Small Enterprises of Celaya, Guanajuato, Mexico; analyzing six ethical values: Principles that guide the behavior of its collaborators, fulfillment of legal regulation, fair competition, pricequality fairness, support to the growth of their suppliers and collaborators report corruption acts. With a sample
\end{abstract}

of 87 Small Enterprises, an analysis was performed through contingency and correlational tables using Pearson's Chi-square test. The results show that, regardless of the enterprise considering themselves Socially Responsible or not, they consider the ethical behavior to be an essential element of their management practices, as well as the way their employees should behave; giving a bigger emphasis to the fulfillment of legal regulations and price-quality equity. Even every ethical values used in this research have positive and significant correlation with corporate social responsibility of the independent enterprises considered or not as Socially responsible. 


\section{INTRODUCCIÓN}

La presión del entorno ha hecho que las empresas busquen ser socialmente responsables (ESR), por lo cual es común observar que un sin número de empresas proclaman que son ESR. Pero no basta con declararlo a sus diversos grupos de interés, deben demostrarlo a través de un comportamiento ético para con su capital intelectual, sociedad y medio ambiente, que son dimensiones de la Responsabilidad Social Corporativa (RSC) también llamada Responsabilidad Social Empresarial (RSE).

Una empresa que actúa con un comportamiento ético es aquella que establece valores y actitudes que guían la conducta de todos los que conforman la empresa desde el dueño o socios, directivos, y demás integrantes de la empresa. Situación que tienen muy asumida las empresas grandes, e incluso instalan un departamento de responsabilidad social, para que su capital intelectual desarrolle estrategias y planes de acción socialmente responsables así como medidas correctivas para mantener un comportamiento ético con sus empleados, clientes, proveedores, gobierno, comunidad y medio ambiente.

Lo ideal sería que todo tipo de empresas de cualquier tamaño mantuvieran un comportamiento ético, diseñarán e implantarán estrategias, acciones y medidas correctivas en los tres ejes principales de la responsabilidad social de las empresas (económico, social y medio ambiente). Pero no siempre les es posible, por falta de recursos humanos, técnicos y financieros, situación que en mayor o menor medida sufren las micro, pequeñas y medianas empresas (Mipymes).

Es de gran importancia investigar sobre el comportamiento ético que guardan las Mipymes como parte de ser ESR, pese a las carencias a las que se enfrentan para seguir adelante. Como indica Narváez, Ferrer, Fernández y Senior (2009) las empresas pequeñas y medianas deben asumir la ética como parte de la empresa, en vía a su desarrollo responsable. Por ello en este trabajo se presenta un estudio sobre las pequeñas empresas, de ahí que el objetivo es analizar la influencia del comportamiento ético en las pequeñas empresas de Celaya, Guanajuato, analizando seis valores éticos: principios que guían la conducta de sus colaboradores, cumplimiento con la normatividad legal, competencia leal, equidad en Precio-calidad, apoyo al desarrollo de sus proveedores y colaboradores que denuncian actos de corrupción. La finalidad de este estudio es que sea de utilidad a las empresas, comunidad académica y gobierno, acerca del comportamiento ético de las pequeñas organizaciones de esta región en particular, pues Celaya es uno de los municipios principales del estado de Guanajuato que esta teniendo un desarrollo económico prominente, además de la significativa derrama de inversión extranjera que esta recibiendo, lo que lo hace una región interesante como objeto de estudio.

Este artículo está estructurado de la siguiente manera: se presenta la revisión bibliográfica sobre los valores éticos que integran el comportamiento ético, objeto de esta investigación, seguido de la metodología para este estudio, posteriormente se muestran los resultados y discusión, para continuar con las conclusiones y referencias bibliográficas.

\section{REVISIÓN BIBLIOGRAFÍCA}

\subsection{Pequeñas empresas}

Las pequeñas empresas son un detónate económico de cualquier economía. En México se pueden clasificarse por sector y número de colaboradores, ventas anuales y tope máximo combinado, de acuerdo al Diario Oficial de la Federación (2009), las pequeñas empresas se 
clasifican en industrias, comercios y servicios con un rango de colaboradores entre 11 a 50, rango en ventas anuales de $\$ 4.01$ hasta $\$ 100$ millones como se muestra en la tabla 1.

Tabla 1: Clasificación de las Pequeñas empresas

\begin{tabular}{lccc}
\hline Sector & Rango de número de trabajadores & $\begin{array}{c}\text { Rango de monto de } \\
\text { ventas anuales (mdp) }\end{array}$ & $\begin{array}{c}\text { Tope máximo } \\
\text { combinado* }\end{array}$ \\
\hline Comercio & Desde 11 hasta 30 & $\$ 4.01$ hasta 100 & 93 \\
Industria y Servicio & Desde 11 hasta 50 & $\$ 4.01$ hasta 100 & 95 \\
\hline
\end{tabular}

*(Número de trabajadores X 10\%) + (Ventas Anuales X 90\%). Fuente: Diario Oficial de la Federación del 30 de junio de 2009.

Otra clasificación es por la edad de la empresa, de acuerdo con INEGI (2014), las empresas se pueden clasificar en empresas de reciente creación (de 0 a 2 años), jóvenes (de 3 a 5 años), adultas (de 6 a 10 años) y mayores (de más de 10 años). Por otro lado el Directorio Estadístico Nacional de Unidades Económicas (DENUE) proporciona una serie de información sobre las empresas, a las que denomina como unidades económicas a nivel nacional, estado o municipio (DENUE, 2016). En México, las pequeñas empresas representan el 2\% (INEGI, 2016a), del total de unidades económicas entre 11 a 50 empleados $(248,963)$, de las cuales en Guanajuato se ubican 12,027 pequeñas empresas (INEGI, 2016b). En el estado, Celaya ocupa el tercer lugar en unidades económicas (Secretaria de Desarrollo Económico Sustentable, 2011), con 1,271 empresas pequeñas (INEGI, 2016b).

\subsection{Responsabilidad Social Corporativa y la ética}

Existen diversas definiciones de Responsabilidad Social Corporativa (RSC), emitidas por diferentes organismos, institutos e investigadores internacionales y nacionales como: AlvaradoHerrera et al. (2017), Links et al. (2017), Business for Social Responsability (2015), Forética (2015), Economistas sin Fronteras (2012), Libro Blanco (2011), ISO 26000 (2010), Ágora Social (2009), CEMEFI (2008), García y Zabala (2008), Comisión Europea (2007), Instituto Ethos Brasil (ETHOS Y CEBRAE, 2007), Marín (2007), Global Reporting Initiative (2006), McWilliams, Siegel y Wright (2006), Vives (2004), Correa (2004), Regil (2003), Libro verde (2001). En las cuales coinciden tres elementos que se deben de incluir en una definición de responsabilidad social: económico, sociedad y medio ambiente.

A la Responsabilidad Social Corporativa (RSC) también conocida como Responsabilidad Social Empresarial (RSE) se pude definir como "una visión de negocios que integra el respeto por las personas, los valores éticos, la comunidad y el medioambiente con la gestión misma de la empresa, independientemente de los productos o servicios que ésta ofrece, del sector al que pertenece, de su tamaño o nacionalidad" (CEMEFI, 2008:2), o bien es una "cultura de negocios basada en principios éticos y firme cumplimiento de la ley, respetuosa de las personas, familias, comunidades y medio ambiente, que contribuye a la competitividad de las empresas, bienestar general y desarrollo sostenible del país" (centraRSE, 2016:1).

Un aspecto que prevalece en estas definiciones es la ética (centraRSE, 2016, CEMEFI, 2008), considerada como parte de la responsabilidad social de la empresa (Kaymak y Bektas, 2017; centraRSE, 2016; Business for Social Responsability, 2015; Forética, 2015; Economistas sin Fronteras, 2012; Libro Blanco, 2011; ISO 26000, 2010; Ágora Social, 2009; Cajiga, 2008; CEMEFI, 2008; García y Zabala, 2008; Comisión Europea, 2007; ETHOS Y CEBRAE, 2007; Libro verde, 2001). Para Perdiguero y García (2005), Perdiguero (2003), Betancur, (2002), Epstein, y Mantilla, (2000), la ética es una de las cuatro características de la responsabilidad empresarial, las otras son la 
económica, legal y discrecionales. En relación a la ética, mismo Perdiguero (2003), indica que esta comprende, estándares y normas acerca de las expectativas de los empleados, dueños o accionistas, consumidores y comunidad en general.

Pudiendo establecer que la ética es un elemento esencial en el desarrollo de ser ESR independientemente del tamaño, sector, actividad de la empresa y en cualquier país o área geográfica donde se establezcan.

\subsection{Comportamiento ético}

La ética es un elemento muy importante tanto para las empresas como para las personas que colaboran en ella, ambas deben de conducir sus actividades con valores éticos, en este sentido Robbins (2004), indica que altos estándares éticos en la empresa, influirán en el comportamiento del personal, por su parte Collier y Esteban (2007) mencionan que una empresa no solo es responsable de su conducta ética, sino de sus impactos en el presente y en el futuro, responsable de motivar a sus empleados a tomar decisiones éticas e inclusive señalan que los empleados con valores y visión ética son los que están alineados a una organización socialmente responsable (SR). En un sentido más amplio es importante que el comportamiento empresarial debe incluir la ética, filantropía, rendición de cuentas, producto, normativa regulatoria y compromisos comerciales (Gardberg, 2017; Nuñez, 2003), así como lo derechos universales, derechos humanos, desarrollo sustentable, en beneficio de un bien común (Chomali y Majluf, 2007). Además, es esencial considerar que un comportamiento ético en las empresas construye una reputación ética beneficiosa para la RSE (Velamuri et al., 2017).

En relación al comportamiento ético desde la perspectiva de la RSC o RSE se puede definir como la gestión empresarial con valores éticos, de ahí que la ética sea parte de la estrategia de la empresa. De acuerdo con Martínez (2005) es conducirse con valores éticos incrustados en las estrategias de la empresa cuidando el beneficio económico, social y medioambiental, elementos sustanciales de la responsabilidad social de la empresa. En este sentido se pronuncia CEMEFI (2008), indicando que una ESR debe de incluir valores éticos como parte de su cultura y estrategia. Lo que conlleva a ventajas con sus Stakeholders como: la confianza, reputación y legitimidad (Muñoz-Martín, 2013; Argandoña, 2007; Sen, 2003), además de reducir costes internos-externos, y aumenta el rendimiento empresarial (Muñoz-Martín, 2013).

Aunque algunos estudios sugieren que la ética esta disminuyendo en las empresas, como el informe de Forética 2011, que indica que solo $16.5 \%$ aplican un código ético de conducta y $9.5 \%$ piensan tenerlo en el futuro, 38\% implementan planes de formación a su personal en la RS y/o ética, mientras que $15.1 \%$ lo hará en el futuro, solo el $5.7 \%$ considera a la ética como una prioridad de desarrollo en RS. Manifestando en el mismo informe que $31.04 \%$ de las pequeñas empresas están abandonando sus actividades de RSE, aunque no menciona cuales. Y en el informe 2015, solo manifiesta que los principios de gestión ética, deben aplicarlos e incluirlos en su estrategia las pymes (Forética, 2015). Por su parte Moreno y Graterol (2011), en su estudio revela que solo $16 \%$ de las pymes adhieren valores (sobre una muestra de 203 Pymes del sector metalmecánico de Venezuela).

\subsection{La Responsabilidad Social en la Pequeñas empresas y su comportamiento ético}

Hablar de responsabilidad social empresarial y comportamiento ético (CE) empresarial, como dos términos aislados, no es posible, porque el CE es una pieza fundamental de la RSE. En este sentido se pronuncian organismos como Forética (2015), quién asevera que el comportamiento ético es un aspecto importante de la RSE, así como Business for Social Responsibility (2008) e ISO 26000 (2010). 
En el ámbito de las Pequeñas y medianas empresas (Pymes), Moreno y Graterol (2011:182) aseguran que algunas Pymes han "plasmado sus valores en un código de ética... que se traducen en una declaración de buenas prácticas empresariales", aunque Fernández (2006), indica que la ética a parte de plasmarla en un código debe llevarse a la acción, y Puerta (2012), señala la importancia de que las empresas tengan un solido comportamiento ético el cual les ayudara a tomar decisiones correctas en época de crisis. Aunque para las empresas constituya un dilema elegir entre el comportamiento ético y obtener el máximo de benéficos (Hovring, 2017), ponderen y decidan gestionar su empresa con comportamiento ético.

Es entonces que ser empresa socialmente responsable, no tiene que ver con el tamaño del negocio, sino con querer serlo, por tanto las pequeñas empresas pueden mostrar un alto compromiso ético. El cual fomentan en sus colaboradores, practicando con ellos la ética y la equidad, así como con sus clientes, proveedores y comunidad (Ríos, López y Contreras, 2013).

\subsection{Elementos del comportamiento ético empresarial}

Son diversos los elementos que componen el comportamiento ético, los cuales pueden ser: principios que guían la conducta de sus colaboradores; cumplimiento con la normatividad legal; competencia leal; equidad en Precio-calidad; apoyo al desarrollo de sus proveedores; y colaboradores denuncian actos de corrupción. A continuación se explica cada uno:

Principios que guían la conducta de sus colaboradores, constituido por valores y actitudes como el respeto, honestidad, compromiso, cooperación, lealtad, confianza, integridad, transparencia en la comunicación, entre otros. Valores y actitudes que guían la conducta de las personas que laboran en la empresa. En este sentido se pronuncia Cajiga (2008), indicando como valores: la honradez, la integridad, la responsabilidad y la transparencia, al igual que ISO 26000 (2010) y Business for Social Responsibility (2008). Por su parte Narváez et al. (2009:115), indican que esta integrada por valores como "la cooperación, responsabilidad, calidad, confianza, seguridad, comunicación, confiabilidad, respeto". O Carroll y Buchholtz (2009), que se pronuncia por una conducta moral, como principio de lo correcto e incorrecto. Y ETHOS (2007), cuestiona a las empresas sobre sus valores. Por ello es necesario que la empresa establezca explícitamente valores fundamentales que guíen el desarrollo de sus estrategias (Waddock y Bodwell, 2017) y la conducta de sus colaboradores.

Cumplimiento con la normatividad legal, es cuando la empresa cumple con todas las normas y obligaciones que imponen las leyes. Cajiga (2008), en su calidad de organismo certificador, en la valoración si otorga o no a las empresas el distintivo de ESR, exige respeto al marco legal y lo observa como un valor empresarial. Mientras que ETHOS (2007:16), en las evaluaciones a empresas sobre la RS realiza un cuestionamiento sobre el "cumplimiento de las leyes y pago de tributos". En cuanto a los trabajos de investigación, en la literatura se encontró que en el instrumento de Zahira y Graterol (2011:187), establecen un rubro sobre las “Prácticas de RSE de acuerdo con leyes". Por su parte Boza y Pérez (2009), manifiestan que uno de los compromisos de la RSC es la parte legal, al igual que Carroll (1991), quien lo maneja como un componente de la RSC, el cual las empresas deben cumplir de manera adecuada con la Ley (Silos, Trujillo y Berruga, 2017; Nuñez, 2003).

Competencia leal, es el posicionamiento responsable ante los competidores. De acuerdo a Cajiga (2008), la competitividad se debe generar a través de confianza. Mientras que en la evaluación de RSE que realiza ETHOS (2007), tiene el indicador denominado como relaciones con la competencia, la cual indica que debe ser leal. Mayormente que en la actualidad la competencia esta creciendo, sugiere mayor responsabilidad social, siendo importante la competencia leal (Ś migielska y Oczkowska, 2017), como uno de los valores de la empresa. 
Equidad en Precio-calidad, es cuando la empresa promueve el equilibrio entre el precio y calidad de los productos y servicios que ofrece a sus clientes. En este sentido Narváez et al. (2009) y Cajiga (2008), señalan que los precios de sus productos y servicios deben ser competitivos, por su parte ETHOS (2007), destaca que uno de los indicadores son las relaciones con la competencia y en ella una variable es el precio, y en la relación con el mercado se cumpla con la ley y el posicionamiento leal. Mientras que para Silos et al. (2017:14) señalan que la relación preciocalidad, contribuye a "generar mayor impacto social y ambiental".

Apoyo al desarrollo de sus proveedores, son los criterios de negociación que permiten el crecimiento futuro de sus proveedores. En este sentido Boza y Pérez (2009), indican que uno de los principios de la RSC es mejorar su relación con sus proveedores. Por su parte ETHOS (2007), contempla el compromiso con los proveedores como un indicador de relaciones con la competencia. Por su parte Silos et al. (2017), señalan el compromiso y responsabilidad en las operaciones con sus proveedores. No obstante las empresas deben de exigir a sus proveedores un comportamiento correcto ante sus colaboradores y clientes, y en este sentido se pronuncia Cajiga (2008) pero no establece el apoyo que la empresa debe brindar al crecimiento de sus proveedores.

Colaboradores que denuncian actos de corrupción, es cuando el personal de la empresa práctica la cultura de la denuncia por sucesos de corrupción dentro y fuera de la empresa. En este sentido Cajiga (2008:34), no es explicito con respecto a los colaboradores, pero señala que "las empresas deberán trabajar contra la corrupción en todas sus formas, incluyendo la extorsión y el soborno... fortalecer los valores fundamentales del estado de derecho, la honradez, la responsabilidad, la integridad y la transparencia". Por su parte ETHOS (2007: 16 y 67), realizan varios cuestionamientos en: su código de conducta "Corrupción activa y pasiva", e incluso tiene un indicador sobre prácticas anticorrupción. Siendo importante trabajar en la anticorrupción en las empresas, colaboradores de las empresas y gobierno, como lo manifiesta Maguiña (2017), la ética en el gobierno y en la formación de sus funcionarios, concientiza en el impacto de la corrupción. Y los colaboradores de las organizaciones son una fuente directa en las denuncias internas y externas Vandekerckhove y Myers (2016), incluso de las instituciones públicas.

\section{METODOLOGÍA}

La metodología utilizada es de tipo descriptivo y correlacional. Realizando un análisis individual descriptivo de cada uno de los ítems que conforman el comportamiento ético tratados en esta investigación tanto de las pequeñas empresas que se consideran responsables como de las que no se consideran ESR, utilizando tablas de contingencia y realizando un análisis de correlaciones a través de ch-cuadrado de Pearson. En base al objetivo de esta investigación se plantea la siguiente hipótesis:

H1: Existe una relación positiva y significativa entre el comportamiento ético y la responsabilidad social de las pequeñas empresas, independientemente de si se consideran ESR o No ESR.

\subsection{Variables del comportamiento ético}

En este estudio se analiza el "comportamiento ético" integrado por seis cuestionamientos a escala Likert 5 puntos: Totalmente en desacuerdo, en desacuerdo, indiferente, de acuerdo, totalmente de acuerdo y la variable de control Responsabilidad Social de la Empresa, bajo la misma escala (ver tabla 2).

Tabla 2: Variables del comportamiento ético 


\begin{tabular}{|c|c|c|c|}
\hline Variable & Indicadores & Código & Definición \\
\hline \multirow{6}{*}{$\begin{array}{l}\text { Comportamiento } \\
\text { ético }\end{array}$} & $\begin{array}{l}\text { Principios que guían la } \\
\text { conducta de sus } \\
\text { colaboradores }\end{array}$ & PGC & $\begin{array}{l}\text { Constituido por valores y actitudes como el respeto, } \\
\text { honestidad, compromiso, cooperación, lealtad, } \\
\text { confianza, integridad, transparencia, cooperación. }\end{array}$ \\
\hline & $\begin{array}{l}\text { Cumplimiento con la } \\
\text { normatividad legal }\end{array}$ & CNL & $\begin{array}{l}\text { Cumplimiento con todas las normas y obligaciones } \\
\text { que imponen las leyes. }\end{array}$ \\
\hline & Competencia leal & $\mathrm{CL}$ & Posicionamiento responsable ante los competidores \\
\hline & Equidad en Precio-calidad & EP-C & $\begin{array}{l}\text { Equilibrio entre el valor económico que asigna a los } \\
\text { productos y servicios y la calidad que ofrece a sus } \\
\text { clientes. }\end{array}$ \\
\hline & $\begin{array}{l}\text { Apoyo al desarrollo de sus } \\
\text { proveedores }\end{array}$ & A enDP & $\begin{array}{l}\text { Criterios de negociación que permiten el } \\
\text { crecimiento futuro de sus proveedores }\end{array}$ \\
\hline & $\begin{array}{l}\text { Colaboradores que } \\
\text { denuncian actos de } \\
\text { corrupción }\end{array}$ & $\begin{array}{l}\text { C que } \\
\text { DC }\end{array}$ & $\begin{array}{l}\text { Personal de la empresa que práctica la cultura de la } \\
\text { denuncia por sucesos de corrupción dentro y fuera } \\
\text { de la empresa. }\end{array}$ \\
\hline \multirow[b]{2}{*}{$\begin{array}{l}\text { Responsabilidad } \\
\text { Social de la Empresa }\end{array}$} & $\begin{array}{l}\text { Empresa Socialmente } \\
\text { Responsable }\end{array}$ & ESR & $\begin{array}{l}\text { Es un ente económico que se conduce bajo un marco } \\
\text { de respecto, con principios éticos con sus } \\
\text { colaboradores, clientes, proveedores, comunidad y } \\
\text { la ley, bajo aspectos económicos, sociales y cuidado } \\
\text { con el medio ambiente. }\end{array}$ \\
\hline & $\begin{array}{l}\text { Empresa con } \\
\text { comportamiento ético que } \\
\text { No se considera Socialmente } \\
\text { Responsable }\end{array}$ & $\begin{array}{l}\text { N son } \\
\text { ESR }\end{array}$ & $\begin{array}{l}\text { Es un ente económico que No se considera } \\
\text { socialmente responsable, pero que tiene principios } \\
\text { que guían la conducta de sus colaboradores, se } \\
\text { conducen con principio éticos con sus clientes, } \\
\text { proveedores y socios comerciales. }\end{array}$ \\
\hline
\end{tabular}

\subsection{Muestra}

Fuente: elaboración propia

La muestra incluye 87 pequeñas empresas ubicadas en Celaya, Guanajuato, de las cuales el $30 \%$ son del sector industrial, $32 \%$ comercial y $38 \%$ servicios (véase tabla 3). La participación corresponde a diversos giros: transporte (1.1\%), outsourcing de personal (1.1\%), imprenta (2.3\%), construcción (14.9\%), funeraria (1.1\%), purificación de agua (1.1\%), educación (2.3\%), instalación y mantenimiento industrial (1.1\%), despacho contable (1.1\%), fabricación de productos prefabricados (1.1\%), consultoría (3.4\%), gasolinera (1.1\%), spa y estética (1.1\%), publicidad (1.1\%), diseño e instalación de sistemas de riego (1.1\%), asesoría especialistas en Pymes (1.1\%), alimentos (2.3\%), hojalatería y pintura (1.1\%), calzado (1.1\%), ganadería (1.1\%), hotelería (2.3\%), servicios administrativos (1.1\%), restaurant (1.1\%), servicio automotriz (1.1\%), serigrafía y diseño (2.3\%), distribuidor de medicamentos, farmacia y droguería, perforación de pozos, fumigación, banquetes, agrícola, turismo, hospital, aseguradoras, y otras empresas comerciales (27.6\%), servicios (2.3\%) e industria (5.7\%) que no quisieron especificar su actividad, además de $13.3 \%$ que no quiso responder.

Tabla 3: Distribución de la muestra

\begin{tabular}{ll}
\hline Sector & Porcentaje \\
\hline Industrial & $30 \%$ \\
Comercial & $32 \%$ \\
Servicios & $38 \%$ \\
Total & $100 \%$ \\
\hline
\end{tabular}

Fuente: elaboración propia

\subsection{Instrumento}

El instrumento original esta dividido en 3 secciones: Datos relacionados con el encuestado, datos generales de la empresa y en la última sección, responsabilidad social de la empresa, la cual 
consta de 12 bloques con un total de 59 ítems, todos a escala Likert. Pero en esta comunicación solo se analizan los ítems relacionados con el bloque comportamiento ético (6): principios que guían la conducta de sus colaboradores, cumplimiento con la normatividad legal, competencia leal, equidad en Precio-calidad, apoyo al desarrollo de sus proveedores y colaboradores denuncian actos de corrupción interna y externa. Y la variable de control "si la empresa participante se considera: Empresa Socialmente Responsable o No es Socialmente Responsable (ESR/N son ESR)", a la que se denomina "Responsabilidad Social de la Empresa". Además de las siguientes variables sociodemográficas: sector, giro, y tamaño de la empresa (correspondientes a la segunda sección) y de la primera sección solo se considero: edad de la empresa.

\subsection{Fiabilidad}

Considerando las dimensiones objeto de este estudio: Comportamiento ético y Empresa Socialmente responsable, se obtiene una fiabilidad de $\alpha=0.819$, denotando la consistencia de los ítems, objeto de este análisis.

\section{RESULTADOS Y DISCUSIÓN}

En este estudio se evalúa si el comportamiento ético influye en las empresas independientemente si se considera o no Empresa Socialmente Responsable (ESR/ N son ESR). Considerando en esta investigación que el comportamiento ético esta compuesto por: 1) principios que guían la conducta de sus colaboradores, 2) cumplimiento con la normatividad legal, 3) competencia leal, 4) equidad de precio-calidad, 5) apoyo al desarrollo de sus proveedores, y 6) colaboradores denuncian actos de corrupción interna y externa.

\subsection{Pequeñas empresas Socialmente Responsables}

Primero se determina que porcentaje de las pequeñas empresas encuestadas se consideran socialmente responsables (84\%) y cuales no (16\%). En la gráfica 1 , se observa que las que $\mathrm{N}$ son ESR, mayormente son empresas de reciente creación (31\%), seguida de empresas jóvenes (6\%) y finalmente el $1 \%$ son empresas adultas. Mientras que el $37 \%$ de las consideran ESR son empresas jóvenes, 31\% empresas de reciente creación, $9 \%$ adultas y $7 \%$ son empresas mayores.

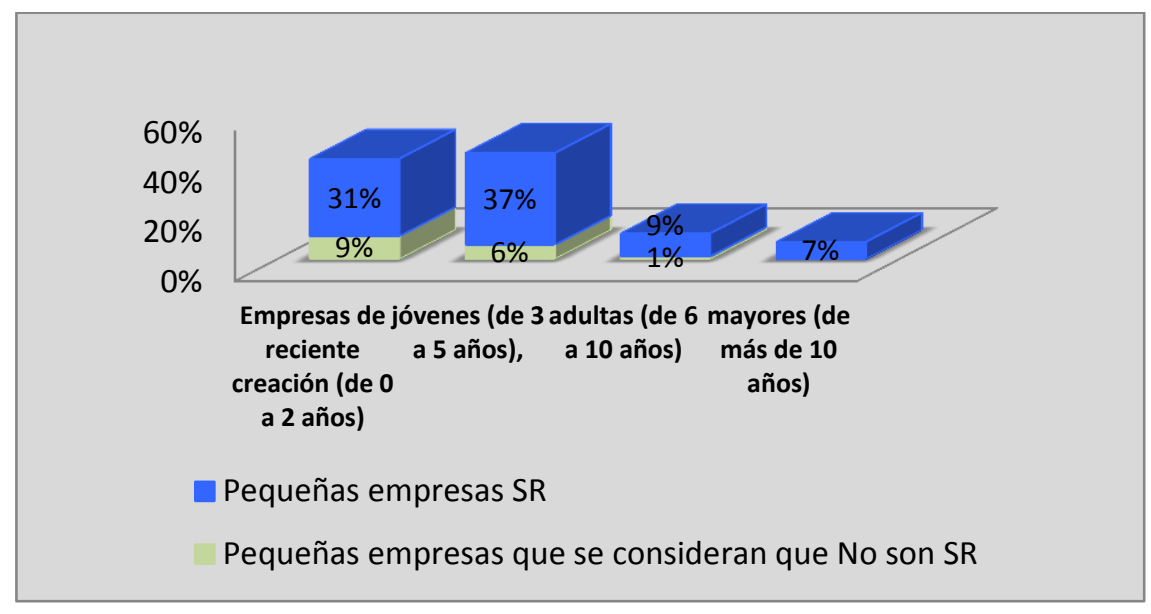

Gráfica 1: Responsabilidad social de las pequeñas empresas en Celaya, Gto. Fuente: elaboración propia 


\subsection{Principios que guían la conducta de sus colaboradores}

El primer elemento considerado en el comportamiento ético de las empresas es el establecimiento de principios que guíen la conducta de sus colaboradores (PGC) como valores, compromiso, honestidad, lealtad, entre otros. En la tabla 4, se muestra que $75.9 \%$ (34.5\% de acuerdo y $41.4 \%$ totalmente de acuerdo) de las empresas que se consideran SR, establecen principios éticos como valores y actitudes como la honestidad, compromiso, lealtad, entre otros, que guían la conducta de sus colaboradores. Mientras que del $16 \%$ de las empresas que No se consideran SR, el $14.9 \%$ establecen principios éticos. Determinando de manera contundente que independientemente si son ESR o no, consideran importante tener un código de conducta ético de sus colaboradores (90.8\%). Por sector se observa de manera general que son las empresas de servicios las que le otorgan mayor importancia a que sus empleados se conduzcan con principios éticos (34.4\%).

Tabla 4: Principios que guían la conducta de sus colaboradores por sector

\begin{tabular}{|c|c|c|c|c|c|c|}
\hline $\begin{array}{l}\text { Pequeñas } \\
\text { empresas }\end{array}$ & Sector & $\begin{array}{l}\text { Completamente } \\
\text { en desacuerdo }\end{array}$ & Neutral & De acuerdo & $\begin{array}{c}\text { Completamente } \\
\text { de acuerdo }\end{array}$ & Total \\
\hline \multirow{4}{*}{ No son ESR $16 \%$} & Industrial & $0.0 \%$ & $0.0 \%$ & $1.1 \%$ & $2.3 \%$ & $3.4 \%$ \\
\hline & Comercial & $0.0 \%$ & $0.0 \%$ & $2.3 \%$ & $2.3 \%$ & $4.6 \%$ \\
\hline & Servicios & $1.1 \%$ & $0.0 \%$ & $3.4 \%$ & $3.4 \%$ & $8.0 \%$ \\
\hline & Total & $1.1 \%$ & $0.0 \%$ & $6.9 \%$ & $8.0 \%$ & $16.0 \%$ \\
\hline \multirow{4}{*}{ ESR $84 \%$} & Industrial & $0.0 \%$ & $3.4 \%$ & $11.5 \%$ & $11.5 \%$ & $26.4 \%$ \\
\hline & Comercial & $0.0 \%$ & $2.3 \%$ & $13.8 \%$ & $11.5 \%$ & $27.6 \%$ \\
\hline & Servicios & $1.1 \%$ & $1.1 \%$ & $9.2 \%$ & $18.4 \%$ & $29.9 \%$ \\
\hline & Total & $1.1 \%$ & $6.9 \%$ & $34.5 \%$ & $41.4 \%$ & $83.9 \%$ \\
\hline \multirow{4}{*}{ Total por Sector } & Industrial & $0.0 \%$ & $3.4 \%$ & $12.6 \%$ & $13.8 \%$ & $29.9 \%$ \\
\hline & Comercial & $0.0 \%$ & $2.3 \%$ & $16.1 \%$ & $13.8 \%$ & $32.2 \%$ \\
\hline & Servicios & $2.3 \%$ & $1.1 \%$ & $12.6 \%$ & $21.8 \%$ & $37.9 \%$ \\
\hline & Total & $2.3 \%$ & $6.9 \%$ & $41.4 \%$ & $49.4 \%$ & $100.0 \%$ \\
\hline
\end{tabular}

\subsection{Cumplimiento con la normatividad legal}

Fuente: elaboración propia

Un aspecto relevante en el comportamiento ético de las empresas es que cumpla con la normatividad legal (CNL). En este sentido en la Tabla 5, se observa de manera general que el $94.2 \%$ de las empresas pequeñas en la ciudad de Celaya, cumplen con la ley, aunque el $3.4 \%$ de las empresas no están de acuerdo con cumplir los mandatos legales o la ignoran (2.3\%). Por sector, los resultados son muy similares en el cumplimiento con los mandatos de la ley, $29.9 \%$ de la industria, $29.8 \%$ del sector comercial, siendo las empresas de servicios, las que mayormente se preocupan por cumplir con la normatividad legal (34.5\%).

Tabla 5: Cumplimiento con la normatividad legal por sector

\begin{tabular}{|c|c|c|c|c|c|c|c|}
\hline $\begin{array}{l}\text { Pequeñas } \\
\text { empresas }\end{array}$ & Sector & $\begin{array}{l}\text { Completamente } \\
\text { en desacuerdo }\end{array}$ & En Desacuerdo & Neutral & De acuerdo & $\begin{array}{c}\text { Completamente } \\
\text { de acuerdo }\end{array}$ & Total \\
\hline \multirow{4}{*}{$\begin{array}{l}\text { No son ESR } \\
16 \%\end{array}$} & Industrial & $0.0 \%$ & $0.0 \%$ & $0.0 \%$ & $1.1 \%$ & $2.3 \%$ & $3.4 \%$ \\
\hline & Comercial & $0.0 \%$ & $0.0 \%$ & $1.1 \%$ & $2.3 \%$ & $1.1 \%$ & $4.6 \%$ \\
\hline & Servicios & $1.1 \%$ & $1.1 \%$ & $0.0 \%$ & $3.4 \%$ & $2.3 \%$ & $8.0 \%$ \\
\hline & Total & $1.1 \%$ & $1.1 \%$ & $1.1 \%$ & $6.9 \%$ & $5.7 \%$ & $16.0 \%$ \\
\hline \multirow{3}{*}{ ESR 84\% } & Industrial & $0.0 \%$ & $0.0 \%$ & $0.0 \%$ & $12.6 \%$ & $13.8 \%$ & $26.4 \%$ \\
\hline & Comercial & $0.0 \%$ & $0.0 \%$ & $1.1 \%$ & $12.6 \%$ & $13.8 \%$ & $27.6 \%$ \\
\hline & Servicios & $1.1 \%$ & $0.0 \%$ & $0.0 \%$ & $8.0 \%$ & $20.7 \%$ & $29.9 \%$ \\
\hline
\end{tabular}




\begin{tabular}{lccccccc} 
& Total & $1.1 \%$ & $0.0 \%$ & $1.1 \%$ & $33.3 \%$ & $48.3 \%$ & $83.9 \%$ \\
\hline \multirow{3}{*}{ Total por } & Industrial & $0.0 \%$ & $0.0 \%$ & $0.0 \%$ & $13.8 \%$ & $16.1 \%$ & $29.9 \%$ \\
Sector & Comercial & $0.0 \%$ & $0.0 \%$ & $2.3 \%$ & $14.9 \%$ & $14.9 \%$ \\
& Servicios & $2.3 \%$ & $1.1 \%$ & $0.0 \%$ & $11.5 \%$ & $32.2 \%$ \\
& Total & $2.3 \%$ & $1.1 \%$ & $2.3 \%$ & $40.2 \%$ & $37.9 \%$ \\
\hline
\end{tabular}

\subsection{Competencia leal}

Analizando si las pequeñas organizaciones de la ciudad de Celaya, ejecutan una competencia leal (CL). En la Tabla 6 , se muestra que $2.7 \%$ de las pequeñas ESR no realizan competencia leal, $8.2 \%$ se mantuvo neutral en su respuesta, y $89 \%$, indica buscar posicionarse ante sus competidores de manera leal. Así mismo la mayoría de las que No se consideran SR señalo competir de manera leal $(85.7 \%)$, aunque $7.1 \%$ no se posicionan de manera leal con su competencia o son indiferentes al respecto.

Tabla 6: Competencia leal

\begin{tabular}{ccccccc}
\hline $\begin{array}{c}\text { Pequeñas } \\
\text { empresas }\end{array}$ & Sector & $\begin{array}{c}\text { Completamente } \\
\text { en desacuerdo }\end{array}$ & Neutral & De acuerdo & $\begin{array}{c}\text { Completamente } \\
\text { de acuerdo }\end{array}$ & Total \\
\hline \multirow{4}{*}{ No son ESR } & Industrial & $0.0 \%$ & $7.1 \%$ & $0.0 \%$ & $14.3 \%$ & $21.4 \%$ \\
& Comercial & $0.0 \%$ & $0.0 \%$ & $21.4 \%$ & $7.1 \%$ & $28.6 \%$ \\
& Servicios & $7.1 \%$ & $0.0 \%$ & $35.7 \%$ & $7.1 \%$ & $50.0 \%$ \\
& Total & $7.1 \%$ & $7.1 \%$ & $57.1 \%$ & $28.6 \%$ & $100.0 \%$ \\
\hline \multirow{5}{*}{ ESR } & Industrial & $0.0 \%$ & $4.1 \%$ & $16.4 \%$ & $11.0 \%$ & $31.5 \%$ \\
& Comercial & $1.4 \%$ & $1.4 \%$ & $12.3 \%$ & $17.8 \%$ & $32.9 \%$ \\
& Servicios & $1.4 \%$ & $2.7 \%$ & $11.0 \%$ & $20.5 \%$ & $35.6 \%$ \\
& Total & $2.7 \%$ & $8.2 \%$ & $39.7 \%$ & $49.3 \%$ & $100.0 \%$ \\
\hline
\end{tabular}

\subsection{Equidad en Precio-calidad}

Otro aspecto importante del comportamiento ético de las empresas es la relación equitativa entre precio-calidad de los productos o servicios (EP-C) que ofrece a sus clientes. Es interesante destacar que de las empresas que No son SR, 7.1.\% no le interesa la equidad en precio- calidad, mientas que el $28.6 \%$ se muestra indiferente a este tema, el $50 \%$ está de acuerdo, y solo el $14.3 \%$ contesto de manera rotunda buscar la equidad en precio-calidad. En relación a las ESR, solo una se mostro indiferente en esta relación, siendo del sector servicios, mientras que 98.6\% busca mantener una relación equitativa entre precio-calidad de los productos y servicios que ofrece a sus clientes. Por sector, las empresas SR de servicio 34.3\% (15.1\% más 19.2\%), destacan en la búsqueda del equilibrio en precio-calidad, los sectores de industrial (31.5\%) y comercial (32.9\%) perciben de manera similar la importancia de la equida en precio-calidad de los productos o servicios que ofrecen (ver tabla 7).

Tabla 7: Equidad en Precio-calidad de los productos o servicios

\begin{tabular}{rcccccc}
\hline $\begin{array}{r}\text { Pequeñas } \\
\text { empresas }\end{array}$ & Sector & En desacuerdo & Neutral & De acuerdo & $\begin{array}{c}\text { Completamente } \\
\text { de acuerdo }\end{array}$ & Total \\
\hline \multirow{3}{*}{ No son ESR } & Industrial & $0.0 \%$ & $0.0 \%$ & $14.3 \%$ & $7.1 \%$ & $21.4 \%$ \\
& Comercial & $0.0 \%$ & $14.3 \%$ & $7.1 \%$ & $7.1 \%$ & $28.6 \%$ \\
& Servicios & $7.1 \%$ & $14.3 \%$ & $28.6 \%$ & $0.0 \%$ & $50.0 \%$ \\
& Total & $7.1 \%$ & $28.6 \%$ & $50.0 \%$ & $14.3 \%$ & $100.0 \%$ \\
\hline \multirow{3}{*}{ ESR } & Industrial & $0.0 \%$ & $0.0 \%$ & $19.2 \%$ & $12.3 \%$ & $31.5 \%$ \\
& Comercial & $0.0 \%$ & $0.0 \%$ & $13.7 \%$ & $19.2 \%$ & $32.9 \%$ \\
& Servicios & $0.0 \%$ & $1.4 \%$ & $15.1 \%$ & $19.2 \%$ & $35.6 \%$ \\
& Total & $0.0 \%$ & $1.4 \%$ & $47.9 \%$ & $50.7 \%$ & $100.0 \%$ \\
\hline
\end{tabular}




\subsection{Apoyo al desarrollo de sus proveedores}

Fuente: elaboración propia

A nivel global, $71.3 \%$ de las ESR y $9.1 \%$ de las que No lo son, buscan la negociación que permita el crecimiento de sus proveedores (A en DP). Realizando el análisis por separado, en la Tabla 8, se observa la fuerte disposición que tienen las pequeñas ESR (84.9\%) a efectuar negociaciones que les permita el crecimiento de sus proveedores, y aunque es muy equitativa por sectores esta forma de conducirse, las de servicio son las que propician mayormente una negociación que favorezca el crecimiento de sus proveedores. Mientras que las empresas de Celaya que No se consideran ESR, se observa que poco más de la mitad (57.1\%), apoyan a que sus proveedores de desarrollen, siendo notorio la indiferencia de las empresas industriales (14.3\%) y de servicio (14.3\%) evadiendo aceptar o rechazar dicho apoyo, y $7.1 \%$ no permiten la negociación del crecimiento de sus proveedores o socios comerciales.

Tabla 8: Apoyo al desarrollo de sus proveedores

\begin{tabular}{rlrrrrr}
\hline $\begin{array}{r}\text { Pequeñas } \\
\text { empresas }\end{array}$ & Sector & $\begin{array}{c}\text { Completamente } \\
\text { en desacuerdo }\end{array}$ & \multicolumn{1}{c}{ Neutral } & \multicolumn{2}{c}{ De } & Completamente \\
acuerdo & de acuerdo & \multicolumn{1}{c}{ Total } \\
\hline \multirow{3}{*}{ No son ESR } & Industrial & $0.0 \%$ & $14.3 \%$ & $0.0 \%$ & $7.1 \%$ & $21.4 \%$ \\
& Comercial & $0.0 \%$ & $7.1 \%$ & $14.3 \%$ & $7.1 \%$ & $28.6 \%$ \\
& Servicios & $7.1 \%$ & $14.3 \%$ & $21.4 \%$ & $7.1 \%$ & $50.0 \%$ \\
& Total & $7.1 \%$ & $35.7 \%$ & $35.7 \%$ & $21.4 \%$ & $100.0 \%$ \\
\hline \multirow{5}{*}{ ESR } & Industrial & $0.0 \%$ & $6.8 \%$ & $16.4 \%$ & $8.2 \%$ & $31.5 \%$ \\
& Comercial & $0.0 \%$ & $5.5 \%$ & $9.6 \%$ & $17.8 \%$ & $32.9 \%$ \\
& Servicios & $1.4 \%$ & $1.4 \%$ & $15.1 \%$ & $17.8 \%$ & $35.6 \%$ \\
& Total & $1.4 \%$ & $13.7 \%$ & $41.1 \%$ & $43.8 \%$ & $100.0 \%$ \\
\hline
\end{tabular}

Fuente: elaboración propia

\subsection{Colaboradores que denuncian actos de corrupción}

Más de la mitad de los colaboradores de las pequeñas empresas de Celaya denuncian actos de corrupción (69.4\%), 15.3\% no lo hacen y en la misma proporción se muestran indiferentes a hacerlo. Analizando los resultados por sector y si son o No ESR, en la tabla 9, son los empresarios SR del sector comercial y de servicios (26.8\%, respectivamente) quienes indican mayormente que sus colaboradores denuncian actos de corrupción (C que DC). Siendo el sector industrial que muestra que sus colaboradores no están de acuerdo o se muestran indiferentes a denunciar actos de corrupción (11.3\%), mientras que los que No son SR manifiestan que los colaboradores del sector servicios (28.6\%) son los que mayormente no les interesa denunciar actos de corrupción. Otro resultado relevante es que las empresas que No se consideran SR, son más susceptibles a evadir la responsabilidad de denuncia sobre actos de corrupción $57.1 \%$, contra el $25.4 \%$ de las que se consideran ESR.

Tabla 9: Colaboradores denuncian actos de corrupción dentro y fuera de la empresa

\begin{tabular}{|c|c|c|c|c|c|c|c|}
\hline $\begin{array}{l}\text { Pequeñas } \\
\text { empresas }\end{array}$ & Sector & $\begin{array}{l}\text { Completamente } \\
\text { en desacuerdo }\end{array}$ & $\begin{array}{c}\text { En } \\
\text { Desacuerdo }\end{array}$ & Neutral & De acuerdo & $\begin{array}{c}\text { Completamente } \\
\text { de acuerdo }\end{array}$ & Total \\
\hline \multirow{4}{*}{$\begin{array}{l}\text { No son } \\
\text { ESR }\end{array}$} & Industrial & $0.0 \%$ & $7.1 \%$ & $0.0 \%$ & $7.1 \%$ & $7.1 \%$ & $21.4 \%$ \\
\hline & Comercial & $7.1 \%$ & $7.1 \%$ & $7.1 \%$ & $0.0 \%$ & $7.1 \%$ & $28.6 \%$ \\
\hline & Servicios & $7.1 \%$ & $7.1 \%$ & $14.4 \%$ & $21.4 \%$ & $0.0 \%$ & $50.0 \%$ \\
\hline & Total & $14.3 \%$ & $21.4 \%$ & $21.4 \%$ & $28.6 \%$ & $14.3 \%$ & $100.0 \%$ \\
\hline \multirow{4}{*}{ ESR } & Industrial & $4.2 \%$ & $1.4 \%$ & $5.7 \%$ & $11.3 \%$ & $9.9 \%$ & $32.4 \%$ \\
\hline & Comercial & $0.0 \%$ & $1.4 \%$ & $4.2 \%$ & $16.9 \%$ & $9.9 \%$ & $32.4 \%$ \\
\hline & Servicios & $1.4 \%$ & $2.8 \%$ & $4.2 \%$ & $14.1 \%$ & $12.7 \%$ & $35.2 \%$ \\
\hline & Total & $5.6 \%$ & $5.6 \%$ & $14.1 \%$ & $42.3 \%$ & $32.4 \%$ & $100.0 \%$ \\
\hline
\end{tabular}

Fuente: elaboración propia 


\subsection{Comportamiento ético de las Pequeñas empresas en Celaya}

Las empresas deben asegurar un adecuado comportamiento ético de todos sus miembros, la cual debe regir la toma de decisiones, resolver problemas, crear estrategias y planes de acción. En este sentido, en la Tabla 10, se puede apreciar que las empresas otorgan al cumplimiento legal ( $\bar{x}=4.43$ ) el principal elemento en su comportamiento ético, seguido de la relación equitativa precio-calidad del producto-servicio $(X=4.37)$, seguido de los principios éticos que guían la conducta de los colaboradores $(\bar{x}=4.36)$, siendo la más baja que sus colaboradores denuncien actos de corrupción al obtener una media de $(\bar{x}=3.76)$, es decir, al empresario no se preocupa tanto por que sus colaboradores denuncien actos de corrupción interna y externa.

Tabla 10: Indicadores del comportamiento ético de las pequeñas empresas en Celaya, Gto

\begin{tabular}{lcc}
\hline Indicadores del comportamiento ético & Media & Desv. típ. \\
\hline Principios éticos que guían la conducta de los colaboradores & 4.36 & .807 \\
Cumplimiento con la normatividad legal & 4.43 & .802 \\
Competencia leal & 4.28 & .885 \\
Equidad en Precio-calidad & 4.37 & .649 \\
Apoyo al desarrollo de sus proveedores & 4.16 & .874 \\
Colaboradores denuncian actos de corrupción interna y externa & 3.76 & 1.172 \\
\hline
\end{tabular}

Analizando si el comportamiento ético influye en las pequeñas empresas de Celaya, Guanajuato independientemente si se consideran o no Socialmente Responsables (ESR/N son ESR), se realizó un análisis correlacional de los indicadores que conforman el comportamiento ético de las ESR y aquellas que $\mathrm{N}$ son ESR. Observando en la Tabla 11, que todos los índices tratados en esta investigación se correlacionan positiva y significativamente a $p=0.01$ y $p=0.05$ con las empresa ESR/ N son ESR. Comparando cada uno, es relevante observar la relación entre el índice Principios que guían la conducta de sus colaboradores y la competencia leal $r=0.708$, así como el cumplimiento con la normatividad legal ( $r=0.590)$; La relación entre CNL con CL $(r=0.652)$, así como CNL con A en DP ( $r=0.548)$; CL y A en DP $(r=0.573)$; así como la relación entre Equidad precio y calidad con la responsabilidad social de la empresa $(r=0.522)$.

Tabla 11: Correlación del comportamiento ético de las pequeñas empresas en Celaya, Gto

\begin{tabular}{|c|c|c|c|c|c|c|c|}
\hline & PGC & $\mathrm{CNL}$ & $\mathrm{CL}$ & EP-C & A enDP & C que DC & $\mathrm{ESR} / \mathrm{N}$ son $\mathrm{ESR}$ \\
\hline Principios que guían la conducta de & & & & & & & \\
\hline sus colaboradores (PGC) & 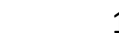 & & & & & & \\
\hline $\begin{array}{l}\text { Cumplimiento con la normatividad } \\
\text { legal }(\mathrm{CNL})\end{array}$ & $.590 * *$ & & & & & & \\
\hline Competencia leal (CL) & $.708 * *$ & $.652 * *$ & & & & & \\
\hline Equidad en Precio-calidad (EP-C) & $.391 * *$ & $.389 * *$ & $.327^{* *}$ & & & & \\
\hline Apoyo al desarrollo de sus & & & & & & & \\
\hline proveedores (A enDP) & $.462 * *$ & $.548 * *$ & $.573 * *$ & $.489 * *$ & & & \\
\hline Colaboradores denuncian actos de & & & & & & & \\
\hline corrupción (C que DC) & $.266^{*}$ & $.311^{* *}$ & $.320 * *$ & $.267^{*}$ & $.233^{*}$ & & 1 \\
\hline Empresas (ESR/ $N$ son ESR) & $.240 *$ & $.430 * *$ & $.291 * *$ & $.522 * *$ & $.374 * *$ & $.316 * *$ & 1 \\
\hline
\end{tabular}

** La correlación significativa al 1\%, * La correlación significativa al 5\%.

Fuente: elaboración propia

En relación al comportamiento ético y RS de las pequeñas empresas de Celaya, Guanajuato, México, la Tabla 12, muestra una relación positiva v significativa, de acuerdo a las pruebas de chicuadrado de Pearson $(p<0.01)$ y la prueba gama $(\ulcorner\gamma=0.554)$, por lo tanto se acepta la $\mathrm{H} 1$, porque el comportamiento ético de las empresas sí influye en que estás sean socialmente responsables.

Tabla 12: Relación entre el comportamiento ético y la Responsabilidad Social de las pequeñas empresas de Celaya, Guanajuato 


\begin{tabular}{lcccc}
\hline \multicolumn{4}{c}{ Estadísticos } \\
\hline Pruebas de chi-cuadrado & Valor & gl & Sig. asintótica (bilateral) \\
\hline Chi-cuadrado de Pearson & $91.432 a$ & 48 & .000 \\
Medidas simétricas & Valor & Error típ. asint. $^{\text {a }}$ & T aproximada $^{\text {b }}$ & Sig. aproximada $^{\text {Sima }}$ \\
\hline Gamma & .554 & .097 & 5.456 & .000 \\
N de casos válidos & 87 & & & \\
\hline
\end{tabular}

a Asumiendo la hipótesis alternativa.

b Empleando el error típico asintótico basado en la hipótesis nula. Fuente: elaboración propia

\section{CONCLUSIONES}

Actualmente, el mundo esta sufriendo de actos de corrupción, siendo imperante que las empresas adopten en su operacionalización y gestión un comportamiento ético. En este sentido el objetivo fue analizar la influencia del comportamiento ético en las pequeñas empresas de Celaya, Guanajuato, México, proporcionando una visión del comportamiento ético analizando seis valores: principios que guían la conducta de sus colaboradores, cumplimiento con la normatividad legal, competencia leal, equidad en Precio-calidad, apoyo al desarrollo de sus proveedores y colaboradores denuncian actos de corrupción. Independientemente de si las pequeñas empresas se consideran o no SR.

En relación a los principios éticos que guían la conducta de los colaboradores de la empresa, es relevante el resultado contundente de que las empresas establecen principios éticos como la honestidad, compromiso, lealtad, valores y actitudes que guían la conducta de sus colaboradores, siendo el sector servicios, quiénes se preocupan más por este aspecto. Sin embargo el énfasis que otorgan las pequeñas empresas en la adopción de principios éticos no ha sido suficiente porque poco más de la mitad de colaboradores de las pequeñas empresas de Celaya denuncian actos de corrupción, siendo preocupante que casi un tercio de los empresarios consideran que sus colaboradores no denuncian actos de corrupción y más allá de este resultado se visualiza que el empresario necesita diseñar estrategias, acciones y medidas que incrementen la ética, pues uno de sus compromisos éticos es concientizar a sus colaboradores la denuncia de actos de corrupción sucedidos dentro y fuera de la organización es importante y se considera dentro de un comportamiento socialmente responsable.

En cuanto al cumplimiento legal, que es otro elemento del comportamiento ético empresarial, las pequeñas empresas de Celaya manifestaron casi en su totalidad cumplir con la normatividad legal, de un modo semejante se preocupan por mantener una equidad en preciocalidad de los productos $y / 0$ servicios que ofrece a sus clientes. Sin embargo un aspecto preocupante es que una de cada diez empresas busca posicionarse ante sus competidores de manera deshonesta.

Es difícil suponer que las pequeñas empresas puedan brindar apoyo al desarrollo de sus proveedores, debido su la falta de recursos humanos, técnicos y financieros y a la preocupación por sostenerse en el mercado. Uno de los resultados más importantes de esta investigación es que el empresario busca que en la negociación con sus proveedores les permita el crecimiento de quiénes les proveen materia prima, o producto terminado, aunque dos de cada diez empresarios no están dispuesto a apoyar a sus proveedores o son indiferentes al respecto.

La evidencia empírica obtenida muestra que independientemente de si la empresa se considera o no socialmente responsable (ESR/N son ESR) visualizan en el comportamiento ético, un elemento indispensable en la forma de gestionar su organización, así como la forma en que deben de conducirse todas las personas que colaboran en ella, representando el cumplimiento legal como el principal elemento en su comportamiento ético, seguido de la relación equitativa preciocalidad de producto y/o servicio, posteriormente los principios éticos que guían la conducta de los colaboradores y apoyo al desarrollo de sus proveedores, mostrando su indiferencia en que sus colaboradores denuncien actos de corrupción interna y externa.

Este artículo contribuye al conocimiento de las empresas, comunidad académica y gobierno, acerca del comportamiento ético de las pequeñas organizaciones de esta región en particular. La limitación del estudio es que se realizó sobre una muestra pequeña, por lo que se sugiere ampliar la muestra al estado de Guanajuato y posteriormente a toda la República mexicana, a fin de determinar resultados a nivel país. De la misma forma en futuras 
investigaciones, se sugiere abordar otros factores que puedan influir en el comportamiento ético empresarial y así indagar el impacto de este en la rentabilidad de la empresa, con la comunidad, en la imagen del organización y en la fidelidad del cliente.

\section{REFERENCIAS}

Ágora Social (2009). Responsabilidad Social Corporativa. ¿Cómo conseguir la colaboración de todos los agentes implicados en el desarrollo?. Ágora Social . Servicios integrales para el tercer sector. Recuperado el 09 de enero de 2016, de http://www.agorasocial.com

Alvarado-Herrera, A; Bigne, E; Aldas-Manzano, J. \& Curras-Perez, R (2017). A Scale for Measuring Consumer Perceptions of Corporate Social Responsibility Following the Sustainable Development Paradigm. Journal of Business Ethics, 140(2), 243-262.

Argandoña, A. (2007). La RS de la empresa a la luz de la ética. Documento de Investigación 708, IESE Business School- Universidad de Navarra. Recuperado de http://www.iese.edu/research/pdfs/di-0708.pdf

Betancur, J. H. (2002). Búsqueda de Espacios Éticos. El Ágora USB, 1(2), 251-230.

Boza, C.J. \& Pérez, E.D. (2009). Estudio de la Responsabilidad Social de las Empresas. Aplicaciones a las empresas de Gran Canaria. Documentos de trabajo en análisis económico. Economic Analysis Working Papers, 8(4), 1-16.

Business for Social Responsibility (2015). Estados Unidos-Códigos de Ética y Entrenamiento Ético. Recuperado el 19 de enero de 2016, de http://www.bsr.org/CSRResources/IssueBriefDetail.cfm?DocumentID=50967

Business For Social Responsibility (2008). BSR/Cone corporate social. Recuperado el 07 de enero de 2016, de http://www.bsr.org/files/BSR_Cone_2008_Survey.pdf

Carroll, A.B. \& Buchholtz A.K. (2009). Business and Society-Ethics and Stakeholder Management, 7 ma Edición, South Western College Publishing, Ohio, U.S.A.

Carroll, A.B. (1991). The Pyramid of Corporate Social Responsibiiity: Toward the Morai Management of Organizational Stakeholders. Business Horizons, 34(4), 39-48.

Cajiga, J.F. (2008). El concepto de Responsabilidad Social Empresarial, CEMEFI. Recuperado el 16 de marzo de 2016, de https://www.cemefi.org/esr/images/stories/pdf/esr/concepto_esr.pdf

CEMEFI (2008). Centro Mexicano para la Filantropía. Recuperado el 02 de enero de 2016, de http://www.cemefi.org/spanish/content/view/1760/25/

CENTRARSE (2016). ¿Qué es la RSE? Recuperado el 05 de enero de 2016, de http://centrarse.org/?page_id=296

Chomali, F. \& Majluf, N. (2007). Ética y Responsabilidad Social en la Empresa. Aguilar Chilena de Ediciones S.A., Santiago. Chile.

Collier, J. \& Esteban, R. (2007). Corporate Social responsibility and employee commitment. Business Ethics A European Review, 16(1), 19-33, DOI: 10.1111/j.1467-8608.2006.00466.x

Comisión Europea (2007). Definición Responsabilidad Social Empresarial. Recuperado el 27 de enero de 2016, de http://www.foro-industrial.com/2007/.../union-europea-recomiendaresponsabilidad-social-corporativa/

Correa, M.E. (2004). Responsabilidad Social Empresarial: una nueva forma de hacer negocios. Revista Futuros, 2(6), 1-6.

DENUE (2016). Directorio Estadístico Nacional de Unidades Económicas. INEGI, Recuperado el 30 de enero de 2016, de http://www3.inegi.org.mx/sistemas/mapa/denue/

ECONOMISTAS SIN FRONTERAS (2012). La RSE ante la crisis. Economista sin Fronteras, Dossieres EsF, 4, Recuperado el 01 de febrero de 2016, de http://plataforma.responsable.net/compartir/rse-crisis

ETHOS (2007). Indicadores Ethos de Responsabilidad Social Empresarial. Instituto ETHOS, Recuperado el 27 de enero de 2016, de https://www3.ethos.org.br/wpcontent/uploads/2013/07/IndicadoresEthos_2013_PORT.pdf

ETHOS, CEBRAE (2007). Empresas \& Impresa: Pauta de responsabilida de Brasil. Ponencia, Primer Foro de Responsabilidad Social Empresarial del MERCOSUR, Recuperado el 20 de enero de 2016, de http://www.scielo.org.mx/scielo.php?script=sci_nlinks\&ref=1699287\&pid=S0186$1042201100030000900004 \&$ Ing=es

Epstein, M. J. \& Mantilla B., S. A. (2000). El desempeño ambiental en la empresa. Prácticas para costear y administrar una estrategia de protección ambiental. ECOE Ediciones.

Fernández, B. (2006). Ética de la dirección: decisión con libertad. México, ediciones Ruz.

Forética (2011). Informe Forética. Evolución de la Responsabilidad Social de las empresas en España 2011. Forética, Recuperado el 17 de enero de 2016, de 
https://www.juntadeandalucia.es/empleo/carl/portal/c/document_library/get_file?uuid=5c3f 1e8f-7b79-45f8-80ce-bdb7a398fe78\&groupld=10128

Forética (2015). Informe Forética 2015 sobre el estado de la RSE en España. Ciudadano consciente, empresas sostenibles. Forética, Recuperado el 01 de enero de 2016, de http://www.foretica.org/informe foretica 2015.pdf

García. J. \& Zabala, H. (2008). Políticas de Estado como sustento de la organización ciudadana. Centro de Investigación de Ciencias Administrativas y Gerenciales. (CICAG), 5, Edición 1.

Gardberg, N; Zyglidopoulos, S; Symeou, P. \& Schepers, D. (2017). The Impact of Corporate Philantrophy on Reputation for Corporate Social Performance. Bussiness and Society, 39, 254288, https://doi.org/10.1177/0007650317694856

George, D. \& Mallery, P. (2003). SPSS for Windows step by step: A simple guide and reference. 11.0 update (4th ed.) Boston: Allyn \& Bacon.

Global Reporting Initiative (2006). Global Reporting Initiative-GRI (2006). A Common Framework for Sustainability Reporting. Recuperado el 20 de enero de 2016, de http://epsu.org/IMG/pdg/Naoko Kubo GRI EUSSWG Process.pdf

Hovring, C.M (2017). Caught in a communicative catch-22? Translating the notion of CSR as Shared value creation in a Danish CSR Frontrunner. Business Ethics A European Review, 26(4), 369381, DOI: $10.1111 /$ beer.12160

INEGI (2014). Micro, pequeña, mediana y gran empresa. Estratificación de los establecimientos, censos económicos 2014. Instituto Nacional de Estadística y Geografía, Recuperado el 09 de septiembre de 2016, de http://internet.contenidos.inegi.org.mx/contenidos/productos//prod_serv/contenidos/espan ol/bvinegi/productos/nueva_estruc/702825077952.pdf

INEGI (2016a). Se difunden estadisticas detalladas sobre las micro, pequeña y medianas empresas del país. Boletín de prensa Núm. 285/16. Recuperado el 20 de diciembre de 2016, de http://www.inegi.org.mx/saladeprensa/boletines/2016/especiales/especiales2016 0702. pdf

INEGIb (2016b). Directorio Estadístico Nacional de Unidades Económicas. Recuperado el 04 de enero de 2016, de http://www3.inegi.org.mx/sistemas/mapa/denue/default.aspx

ISO 26000 (2010). ISO 26000-Social Responsibility. Recuperado el 24 de febrero de 2016, de http://www.iso.org/iso/home/standards/iso26000.htm

LIBRO BLANCO (2011). Libro Blanco de la Responsabilidad Social Corporativa. CEOE CEPYME, Cantabria, Santander, España.

LIBRO VERDE (2001). Fomentar un marco europeo para la responsabilidad social de las empresas. Recuperado el 22 de diciembre de 2015, de http://europa.eu.int/comm/off/green/indexes.ht

Kaymak, T. \& Bektas, E. (2017). Corporate Social Responsibility and Governance: Information Disclosure in Multinational Corporations. Corporate Social Responsibility and Environmental Management. doi: 10.1002/csr.1428

Lins, K. V.. Servaes. H. \& Tamavo. A. (2017). Social Capital. Trust, and Firm Performance: The Value of Corporate Social Responsibility during the Financial Crisis. The Journal of Finance, 72: 17851824. doi:10.1111/jofi.12505

Maguiña, B. A. (2017). Conversatorio «Ética y corrupción en la función pública»: relatoría general. Repositorio académico UPC. Recuperado el 08 de agosto de 2017 de http://hdl.handle.net/10757/621610

Marín, L. (2007). il need you too! Corporate identitiy attractiveness for consumers and the role of Social Responsibility. Journal of Buiness Ethics. 71(3), 245-260.

Martínez, H.H. (2005). El marco ético de la Responsabilidad social empresarial. editorial Pontificia Universidad Javeriana. Colección Biblioteca del Profesional.

McWilliams, A., Siegel, D. \& Wright, D. (2006). Introduction by Guest Editors Corporate Social Responsibility: International Perspectives. Journal of Business Strategies, 23(1), 1-7.

Moreno, Z. \& Graterol, D.( 2011). Prácticas de responsabilidad social empresarial en la Pyme. Un estudio en el sector metalmecánico de Barquisimeto, Estado Lara, Venezuela. Contaduría y Administración, n.235, p.175-194, sep-dic

Muñoz-Martín, J. (2013). Etica empresarial, Responsabilidad Social Corporativa (RSC) y Creación de Valor Compartido (CVC). Revista de Globalización, Competitividad y Gobernabilidad: GCG; Madrid , 7(3), 76-88. Doi: 10.3232/GCG.2013.V7.N3.05

Narváez, M., Ferrer, J., Fernández, G. \& Senior, A. (2009). Rasgos del componente ético en el cumplimiento de la gestión empresarial social de la pequeña y mediana empresa de la región Paraguaná. Revista de Ciencias Sociales RSC. XV(1), 112-125. 
Nuñez, G. (2003). La responsabilidad social corporativa en un marco de desarrollo sostenible. CEPAL, Medio ambiente y desarrollo. Recuperado el 08 de diciembre de 2015 de http://repositorio.cepal.org/bitstream/handle/11362/5779/S0310754_es.pdf;jsessionid=7FFF 3A95B8028E1E1904D84AF3C08525?sequence=1

Perdiguero, T. G. \& García R., A. (2005). La responsabilidad social de las empresas y los nuevos desafíos de la gestión empresarial. Universitat de València, España.

Perdiguero, T.G. (2003). La responsabilidad social de las empresas en un mundo global. Barcelona: Anagrama.

Puerta, J. F. (2012). La RSE en la tormenta perfecta. En De La Cuesta, M., Paredes, J.D. La RSE ante la crisis. Economistas sin Fronteras. Dossieres EsF. n.4.

Regil, C.A. (2003). Responsabilidad Social Corporativa, Disciplina Todavía en Estado de Inocencia. En los Breviarios Temáticos Lisdinys, Alianza Global lus Semper, 1-8, Recuperado el 03 de diciembre de 2015

de http://jussemper.org/Inicio/Resources/RSC\%20estado\%20inocencia.pdf

Ríos, M.M., López, S.A. \& Contreras, S.R. (2013). La responsabilidad social empresarial y la sustentabilidad del Plan de negocios. En Ríos y Ferrer (coordinador), Plan de negocios para pymes, Pearson, México.

Robbins. S. (2004). El Comportamiento Organizacional. México: Pearson Educación. Quinta Edición.

Secretaria de Desarrollo Económico Sustentable (2011). Indicadores Económicos del Municipio de Celaya. Guanajuato, Gobierno del Estado. Recuperado el 02 de enero de 2016 de http://portalsocial.guanajuato.gob.mx/sites/default/files/documentos/2010_SDES_Indicadore s\%20del\%20Municipio\%20de\%20Celaya.pdf, 2011.

Sen, A.(2003). Ética en la empresa y desarrollo económico. En Cortina A. (Ed) Construir confianza. Madrid, Trotta.

Silos, J. Trujillo, R. \& Berruga, B. (2017). La responsabilidad social Corporativa (RSC) en la contratación pública de servicios de seguridade privada. Forética y Aproser, Madrid España. Recuperado el 04 de agosto de 2017, de http://aproser.es/web/wpcontent/uploads/2017/03/RSC_en_contrataci\%C3\%B3n_p\%C3\%BAblica_servicios_seguridad_ privada.pdf

S migielska, G. \& Oczkowska, R. (2017). Retailers' Responsibility towards Consumers and Key Drivers of Their Development in Poland. Administrative sciences, 7(3), 1-15. DOI:10.3390/admsci7010003

Vives, A. (2004). The Role of Multilateral Development Institutions in Fostering Corporate Social Responsibility. 47(3), in Development Department, Technical Papers Series, Washington, D.C.: Inter-American Development Bank.

Vandekerckhove, W. \& Myers, A. (2016). ¿Cuál es el papel de la protección de los informantes en la lucha contra la corrupción y el buen gobierno? En PSI Public Services Internationales (Eds.) Jaque mate a la corrupción: Argumentos a favor de una amplia protección de los informantes, PSI Public Services International. Recuperado el 08 de agosto de 2017 de http://gala.gre.ac.uk/16668/7/16668\%20VANDEKERCKHOVE_Whistleblower_Protection_201 6.pdf

Velamuri, R; Venkataraman, S. \& Harvey, W. (2017). Seizing the Ethical High Ground: Ethical Reputation Building in Corrupt Environments. Journal of Management Studies, 54 (5), 647675. DOI: $10.1111 /$ joms.12248.

Waddock . S. \& Bodwell. C. (2017). Total Responsibility Managemente. The Manual. Taylor \& Francis. New York, U.S.A.

Zahira, M., Graterol, D. (2011). Prácticas de responsabilidad social empresarial en la Pyme. Un estudio en el sector metalmecánico de Barquisimeto, Estado Lara, Venezuela. Contaduría y Administración, 235, 175-194. 